\title{
Crioconservación de semen y calidad espermática en sabaleta Brycon henni (Pisces: Characidae)
}

\author{
Semen cryoconservation and sperm quality regarding \\ the Neotropical fish Brycon henni (Pisces: Characidae)
}

\section{Criopreservação de sêmen e qualidade do esperma da Sabaleta Brycon henni (Pisces: Characidae)}

\author{
Hermes Pineda-Santis ${ }^{1}$; Jorge Gómez-Oquendo ${ }^{2}$; Juan Montoya-Páez ${ }^{3}$; Verónica Toro-Rendón ${ }^{3}$; \\ Oscar Acevedo-Villa ${ }^{4}$, Giovanni Restrepo-Betancur ${ }^{5}$
}

1 Biólogo, MSc; Docente Asociado; Grupo de Investigación Acuícola, Facultad de Ciencias Agrarias, Politécnico Colombiano Jaime Isaza Cadavid, Medellín.

2 MV, Docente Titular; Grupo de Investigación en Biotecnología Animal, Facultad de Ciencias Agrarias, Politécnico Colombiano Jaime Isaza Cadavid, Medellín.

3 Ing. Agr, Profesional Independiente.

4 Biólogo, Esp. Acui, cMSc; Docente Auxiliar; Facultad de Ciencias Agrarias, Politécnico Colombiano Jaime Isaza Cadavid, Medellín.

5 Zoot, MV, MSc, PhD; Docente Asistente; Grupo de Investigación en Biotecnología Animal; Departamento de Producción Animal, Facultad de Ciencias Agrarias, Universidad Nacional de Colombia, Medellín.

Email: hrpineda@elpoli.edu.co

Recibido: noviembre 3 de 2014

Aprobado: julio 23 de 2015

\begin{abstract}
Resumen
La crioconservación de semen es una técnica utilizada tanto en humanos como en animales, permitiendo la congelación del material genético que será utilizado en la reproducción. El objetivo de esta investigación fue evaluar el efecto de tres crioprotectores sobre los parámetros de movilidad y vitalidad en el semen post descongelado de la sabaleta Brycon henni (Pisces: Characidae). Tres crioprotectores: dimetilformamida (DMF), dimetilsulfoxido (DMSO) y etilenglicol (EG), fueron utilizados independientemente para la crioconservación seminal, a una concentración de 5\%, el semen fue empacado en pajillas de $0.5 \mathrm{ml}$. Diecinueve machos sexualmente maduros y 116 pajillas fueron analizadas en el laboratorio de Biotecnología Animal del Politécnico Colombiano Jaime Isaza Cadavid. En la evaluación del semen fresco y post descongelado se utilizó el Sistema de Análisis de Clase $\left(S C A^{\circledR}\right)$, para los parámetros de movilidad total (MT), movilidad progresiva (MP), velocidad curvilínea (VCL), velocidad rectilínea (VSL) y velocidad promedio (VAP), entre otros. La vitalidad espermática post descongelación se evaluó mediante microscopia de fluorescencia utilizando la combinación de sondas SYBR/IP. Para el análisis estadístico se ajustaron modelos lineales generalizados (GLM) y las medias se compararon por la prueba de

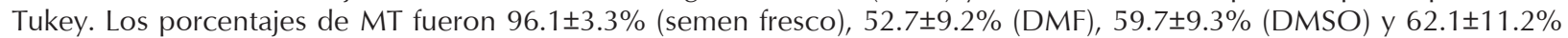
(EG). Mientras que para la MP fueron de 73.3 \pm 14.2 (semen fresco), $11.7 \pm 6.7 \%$ (DMF), $13.8 \pm 6.8 \%$ (DMSO) y $15.6 \pm 7.8 \%$ (EG). No hubo diferencias significativas entre DMSO y EG, pero fueron superiores a DMF $(\mathrm{P} \leq 0.05)$, para ambas variables. VCL, VSL y VAP fueron estadísticamente superiores para EG $(\mathrm{P} \leq 0.05)$. DMSO presentó valores mayores de vitalidad espermática $(82.0 \pm 1.9 \%)$, cuando fue comparado con DMF $(73.5 \pm 5.9 \%)$ y EG $(69.2 \pm 6.7 \%)(P \leq 0.05)$. Se concluye que DMSO
\end{abstract}


y EG presentan porcentajes superiores de MT y MP que DMF, mientras DMSO produce mejores resultados de vitalidad, cuando se evalúa el semen post descongelado de sabaleta Brycon henni.

Palabras claves: Crioprotectores, peces, viabilidad, vitalidad

\begin{abstract}
Semen cryopreservation (i.e. sperm banking) is a technique which is used for both humans and animals; it allows the genetic material which will be used in reproduction to be frozen. This research was aimed at evaluating the effect of three cryoprotectants on the mobility and vitality of post-thaw semen from the trans-Andean, omnivorous fish Brycon henni (Pisces: Characidae). Three cryoprotectants (N,N-dimethylformamide (DMF), dimethyl sulphoxide (DMSO) and ethylene glycol (EG)) were used independently for semen cryopreservation ( $5 \%$ concentration); B. henni semen was packed in 0.5 $\mathrm{ml}$ plastic mini-straws. Nineteen sexually-mature males and 116 straws were analysed at Animal Biotechnology Laboratory of Politécnico Colombiano Jaime Isaza Cadavid. A computer-assisted sperm class analyser (SCA) system was used for evaluating fresh and post-thaw semen regarding the following parameters: total motility (TM), progressive motility (PM), curvilinear velocity (VCL), straight line velocity (VSL) and average path velocity (VAP). Post-thaw sperm vitality was evaluated by fluorescence microscopy using the SYBR Green I/PI probe assay. Statistical analysis involved fitting generalised linear models (GLM) and Tukey multiple comparison test was used for comparing means. TM percentages were $96.1 \pm 3.3 \%$

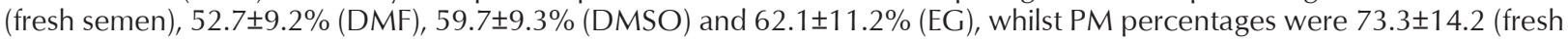
semen), $11.7 \pm 6.7 \%$ (DMF), $13.8 \pm 6.8 \%$ (DMSO) and 15.6 $\pm 7.8 \%$ (EG). There were no statistically significant differences between DMSO and EG, but these were greater than those for DMF ( $\leq \leq 0.05)$ for both variables. Differences for VCL, VSL and VAP were statistically greater for EG $(\mathrm{p} \leq 0.05)$. DMSO had greater sperm vitality values $(82.0 \pm 1.9 \%)$ compared to DMF $(73.5 \pm 5.9 \%)$ and EG $(69.2 \pm 6.7 \%)(p \leq 0.05)$. it was thus concluded that DMSO and EG had greater TM and PM percentages than DMF, whilst DMSO produced better results regarding vitality when evaluating post-thaw semen from the trans-Andean, omnivorous fish Brycon henni.
\end{abstract}

Key words: Cryoprotectant, fish, viability, vitality.

\title{
Resumo
}

A criopreservação do sémen é uma técnica utilizada em humanos e animais, permitindo o congelamento do material genético a ser utilizado na reprodução. O objetivo deste trabalho foi avaliar o efeito de três crioprotetores sobre os parâmetros de mobilidade e vitalidade no sêmen pós-descongelamento da Sabaleta Brycon henni (Pisces: Characidae). Três crioprotectores, dimetilformamida (DMF), dimetilsulfóxido (DMSO) ou etilenoglicol (EG) foram usados independentemente para a criopreservação seminal, a uma concentração de 5\%, o sêmen foi empacotado em canudos de 0.5 ml. Dezenove machos sexualmente maduros e 116 canudos foram analisados no Laboratório de Biotecnologia Animal do Politécnico Colombiano Jaime Isaza Cadavid. Na avaliação da mobilidade do sêmen fresco e após a descongelação, foi usado o Sistema de Análise de Classe $\left(\mathrm{SCA}^{\circledR}\right)$ para os parâmetros de mobilidade total $(\mathrm{MT})$, mobilidade progressiva (MP), velocidade curvilinear (VCL), velocidade em linha reta (VSL) e velocidade média (VAP), entre outros. A viabilidade espermática após o descongelamento foi realizada por meio de microscopia de fluorescência utilizando a combinação de sondas SYBR/ IP. Para a análise estatística, modelos lineares generalizados (GLM) foram ajustados e as médias foram comparadas pelo

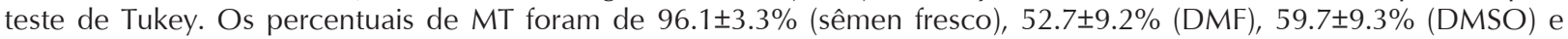
$62.1 \pm 11.2 \%$ (EG). Enquanto que para o MP foram $73.3 \pm 14.2$ (sêmen fresco), $11.7 \pm 6.7 \%$ (DMF), 13.8 $\pm 6.8 \%$ (DMSO) e $15.6 \pm 7.8 \%$ (EG). Não houve diferenças significativas entre DMSO e EG, mas foram superiores ao DMF (P $\leq 0.05)$ para as

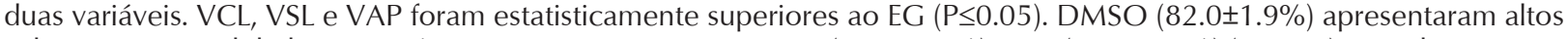

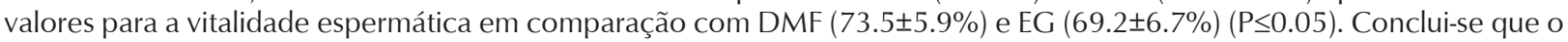
DMSO e EG têm maiores percentuais de MT e MP que DMF, enquanto DMSO produz melhores resultados da vitalidade, quando o sêmen após o descongelado da Sabaleta Brycon henni e avaliado.

Palavras chave: Crioprotetores, peixes, viabilidade, vitalidade.

\section{Introducción}

El uso de la crioconservación de semen en peces se ha desarrollado con éxito en numerosas especies alrededor del mundo (Tiersch, 2008), incluyendo varias especies nativas de gran interés comercial en Colombia como Piaractus brachypomus (Fresneda et al., 2004; Navarro et al., 2004; González et al., 2011), Brycon amazonicus (Pardo-Carrasco et al., 2006; Cruz-Casallas et al., 2006), Pseudoplatystoma fasciatum (Pinzón-Arciniegas et al., 2005; Ramírez et al., 2008), Brycon moo- rei (Betancur et al., 2009), Sorubim cuspicaudus (Reza y Salas, 2010), Prochilodus magdalenae (Martínez et al., 2011; Martínez et al., 2012) y Colossoma macropomum (Varela-Junior et al., 2012).

La crioconservación ha facilitado la reproducción artificial en peces, debido a que mantiene la viabilidad del esperma almacenado por largo tiempo en nitrógeno líquido. Bajo esta consideración, sólo se necesita de la colecta de gametos femeninos, luego de la inducción 
de las hembras, para eliminar el problema del asincronismo reproductivo (Viveiros y Godinho, 2009). Así mismo, ha demostrado ser efectivo en los programas de mejoramiento animal, de reproducción segura por fuera de la estación reproductiva y en el movimiento e intercambio de material genético entre productores. Así mismo permite la mejora de la eficiencia en la utilización de los parentales, y en la disminución de la presión sobre las poblaciones silvestres, en procura de nuevos sementales (Medina et al., 2005).

La sabaleta Brycon henni (Eigenmann - 1913) es una especie nativa presente en los pequeños ríos que nacen en la cordillera Central de Colombia (Dahl, 1971). Se deben considerar los múltiples escenarios para los procesos de conservación genética, en donde se han reportado subpoblaciones con distribución por la parte alta, media y baja de los ríos Cauca y Magdalena (Pineda-Santis et al., 2007; Hurtado et al., 2011). Las poblaciones naturales de la sabaleta Brycon henni están decreciendo por la sobrepesca, la contaminación de su hábitat y la fragmentación de las corrientes por la construcción y operación de represas (Lenis et al., 2009). Su apreciado sabor, alto precio y comportamiento agresivo durante la pesca, la convierten en un gran potencial para la acuicultura, la recreación, la propagación artificial y la reconstitución genética con propósitos de conservación y manejo en cautiverio (Montoya et al., 2006).

Para esta especie se han realizado estudios sobre la caracterización seminal y el potencial de membrana (Tabares et al., 2004; Tabares, 2005; Tabares et al., 2006; Tabares et al., 2007), pero no estudios relacionados con los protocolos de crioconservación de semen, lo que hace necesario profundizar en este aspecto. La vitalidad espermática es otro aspecto fundamental en la evaluación espermática, como indicador de la integridad de la membrana plasmática. Mediante las sondas fluorescentes SYBR-14 y yoduro de propidio (PI), es factible evaluar dicha integridad, toda vez que ambos actúan mediante la penetración de la membrana espermática, marcando las células viables y las bombas iónicas funcionales (Guillan et al., 2005). SYBR-14 identifica a todos los espermatozoides en la muestra (vivos y muertos), mientras que el PI solo tiñe los núcleos de los espermatozoides muertos o degenerados, las cuales han perdido la integridad de su membrana y por consiguiente se tiñen de rojo (Guillan et al., 2005; Ramalho-Santos et al., 2007). Por todo lo anterior, se propuso como objetivo de esta investigación, evaluar los efectos de la crioconservación del semen de sabaleta Brycon henni con tres sustancias crioprotectoras: Dimetilformamida (DMF), Dimetilsulfóxido (DMSO) y
Etilenglicol (EG), sobre diferentes parámetros relacionados con la movilidad y la vitalidad espermática.

\section{Materiales y métodos}

\section{Colección de semen}

Todos los peces se manejaron según las directrices publicadas para la experimentación de peces en las Buenas Prácticas en la Producción Acuícola (ICA, 2007). Diecinueve machos sexualmente maduros de la sabaleta Brycon henni se seleccionaron del Centro Experimental y de Producción Acuícola - CEPA (06 26 $37.57^{\prime \prime}$ $\mathrm{N}, 75^{\circ} 44^{\prime} 04.93^{\prime \prime} \mathrm{W}$ ) en San Jerónimo (Antioquia - Colombia), para cinco procesos ocurridos entre los meses de mayo a diciembre, en épocas por fuera y dentro de la temporada de lluvias. Los animales no fueron inducidos hormonalmente, dado que en el periodo de estudio contaron con una aceptable producción seminal. Se sometieron a condiciones iguales de alimentación, ambiente, manejo productivo y reproductivo. Para la colección del semen, los peces se anestesiaron (MS-222 ${ }^{\circledR}$, Western Chemical, Inc.) para evitar el estrés y se colocaron individualmente sobre una toalla húmeda. Se cubrió la cabeza, se retiró el exceso de agua de la zona ventral, alrededor del poro genital y mediante una leve presión cráneo caudal, se procedió a la extracción del semen en un tubo de $1.5 \mathrm{ml}$. La activación espermática se evaluó por microscopía (Eclipse E200, Nikon, Inc), ubicando sobre un portaobjetos, 1 $\mu \mathrm{l}$ de semen y $10 \mu \mathrm{l}$ de agua. Solo se incluyeron en el estudio las muestras en que la mayoría de los espermatozoides respondieron a la activación. Las muestras individuales fueron diluidas en proporción 1:5, en un diluyente a base de leche descremada, azúcares y antibióticos, con una osmolaridad de $315 \pm 10$ Osm $/ \mathrm{Kg}$ (EquiPro ${ }^{\circledR}$, Minitube, Tiefenbach, Alemania). Posteriormente, el semen diluido fue refrigerado $\left(5^{\circ} \mathrm{C}\right)$ y transportado al Laboratorio de Biotecnología Animal en el Centro de Laboratorios del Politécnico Colombiano Jaime Isaza Cadavid, en Bello (Antioquia - Colombia).

\section{Evaluación del semen fresco}

Una vez en el laboratorio, el semen de cada pez se evaluó mediante un sistema de análisis espermático asistido por computador $\left(\mathrm{SCA}^{\circledR}\right.$, Microptic S.L., España). Se utilizó un procedimiento modificado del reportado por Restrepo et al., (2013). Se empleó un microscopio de contraste de fase (Eclipse E200, Nikon, Inc.) con una cámara digital (Scout SCA780, Basler). Se estableció una configuración específica para el software de: cámara de cubreobjetos de $20 \mathrm{~mm}$ por $20 \mathrm{~mm}$, óptica en lente de ph-, gota de $7 \mu \mathrm{L}$, platina tér- 
mica a $37^{\circ} \mathrm{C}$ y un tamaño de partícula de 1 a $20 \mu \mathrm{m}$. En una cámara de neubauer (para evitar el desplazamiento de la muestra) se mezclaron $1 \mu \mathrm{l}$ de semen y $10 \mu \mathrm{l}$ de agua. Una vez ocurrida la activación del semen, se evaluaron los parámetros: movilidad total (MT), movilidad progresiva (MP), velocidad curvilínea (VCL), velocidad lineal (VSL), velocidad media (VAP), índice de linealidad (LIN = VSL / VCL x 100), índice de rectitud $(\mathrm{STR}=\mathrm{VSL} / \mathrm{VAP} \times 100)$, índice de oscilación $(\mathrm{WOB}=$ VAP / VCL x 100), amplitud lateral de la cabeza (ALH) y frecuencia de batida (BCF).

\section{Crioconservación de semen}

Las muestras seminales individuales colectadas en cada proceso, se mezclaron y luego se dividieron en tres alícuotas. Cada alícuota se diluyó en proporción 1:5, en EquiPro ${ }^{\circledR}$ suplementado con $5 \%$ de crioprotector: dimetilformamida (DMF), dimetilsulfoxido (DMSO) o etilenglicol (EG), y $5 \%$ de yema de huevo, para una concentración final aproximada de $1000-2000 \times 10^{6}$ espermatozoides / $\mathrm{ml}$. La mezcla se mantuvo en refrigeración a $4{ }^{\circ} \mathrm{C}$ por 30 min, luego se empacó en pajillas de $0.5 \mathrm{ml}$, las cuales se refrigeraron nuevamente a $4{ }^{\circ} \mathrm{C}$ por $10 \mathrm{~min}$. Posteriormente, se sometieron a crioconservación rápida en vapores de nitrógeno líquido, colocándolas horizontalmente a una distancia de $4 \mathrm{~cm}$ sobre su superficie, durante $10 \mathrm{~min}$, al cabo de los cuales, se sumergieron en un tanque de almacenamiento de nitrógeno líquido a $-196{ }^{\circ} \mathrm{C}$. Después de una semana, se realizó la descongelación de las pajillas en un baño maría $\left(37^{\circ} \mathrm{C} / 30\right.$ seg.), y se realizaron las evaluaciones de los parámetros de movilidad espermática, mediante el sistema $\mathrm{SCA}^{\circledR}$, de acuerdo al procedimiento descrito previamente.

\section{Evaluación de la vitalidad}

La vitalidad espermática (VE) se evaluó mediante el procedimiento descrito por Gamboa et al., (2010), con el kit Live/Dead (Molecular Probes Inc). Una suspensión de espermatozoides en buffer fosfato con $1 \%$ de albúmina sérica bovina (BSA), se incubó por 5 min a $35{ }^{\circ} \mathrm{C}$ con el fluorocromo para ácidos nucleicos SYBR-14 (fluorescencia verde en todos los espermatozoides), a una concentración final de $6 \mathrm{mM}$. Luego los espermatozoides se incubaron de igual forma con el fluorocromo de ácidos nucleicos yoduro de propidio (fluorescencia roja en células muertas) a $0.48 \mathrm{mM}$. Muestras de $5 \mu \mathrm{l}$ se pusieron en un portaobjetos de vidrio, con un cubreobjetos y se contaron 200 células mediante un microscopio E200 con fluorescencia HBO (Nikon, Inc.) a longitudes de onda entre 550 y $595 \mathrm{~nm}$. Se estableció el porcentaje de vitalidad, sien- do considerados como vivos, aquellos espermatozoides que presentaron un patrón de fluorescencia verde. A su vez se consideraron como muertos, aquellos espermatozoides con fluorescencia roja (Guillan et al., 2005; Ramalho-Santos et al., 2007).

\section{Diseño estadístico}

Para la evaluación estadística se realizó un diseño completamente al azar. Los datos fueron analizados mediante modelos lineales generalizados (GLM) para las variables dependientes de calidad seminal.

El modelo empleado fue:

$$
\mathrm{Y}_{i j k l m n}=\mu+\mathrm{T}_{i}+\mathrm{R}_{j}+\mathrm{C}_{k-\mathrm{r}}+\mathrm{e}_{i j k l m n}
$$

donde:

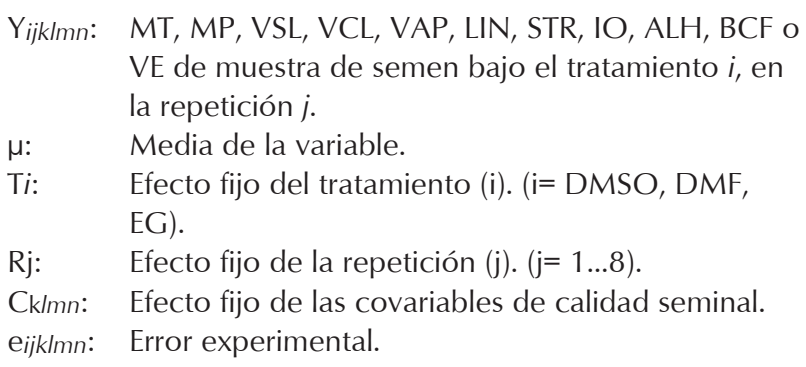

Se realizó un análisis de correlación de Pearson con el fin de definir las covariables. Se comprobó la normalidad de los datos por la prueba K-S. Para la comparación de las medias ajustadas entre los tratamientos, se realizó una prueba de Tukey. Todos los análisis fueron realizados mediante el programa SAS 9.2 (SAS Inst. Inc., Cary, NC).

\section{Resultados}

\section{Semen fresco}

El semen presentó las siguientes características: color blanco, aspecto cremoso, volumen de $500 \pm 220 \mu \mathrm{l} /$ pez y concentración espermática de $14554 \pm 5138$ x $10^{6}$ espermatozoides/ml. Los resultados para las variables de movilidad espermática evaluados mediante el sistema SCA ${ }^{\circledR}$ del semen fresco de la sabaleta Brycon henni, se presentan en la Tabla 1.

\section{Semen criopreservado}

Se realizó la crioconservación y evaluación de 116 pajillas. El efecto del tratamiento (crioprotectores) fue significativo para las variables observadas. El modelo lineal ajustado para las variables presentaron coeficien- 
tes de determinación $\left(\mathrm{R}^{2}\right)$ mayores al 70\%. Todos los coeficientes de correlación (Pearson) fueron altos y positivos $(r>0.7)$. Los resultados de la evaluación del semen post descongelado se presentan en la Tabla 2.

Tabla 1. Resultados de movilidad espermática para semen fresco de la sabaleta Brycon henni, obtenidos mediante el sistema de análisis asistido por computador SCA ${ }^{\circledR}$.

\begin{tabular}{|c|c|}
\hline Parámetro & Media \pm DE \\
\hline MT $(\%)$ & $96.1 \pm 3.3$ \\
\hline MP $(\%)$ & $73.3 \pm 14.2$ \\
\hline VCL $(\mu \mathrm{m} / \mathrm{seg})$ & $115.4 \pm 24.2$ \\
\hline VSL $(\mu \mathrm{m} / \mathrm{seg})$ & $64.8 \pm 15.3$ \\
\hline VAP $(\mu \mathrm{m} / \mathrm{seg})$ & $84.0 \pm 18.9$ \\
\hline LIN $(\%)$ & $55.9 \pm 4.3$ \\
\hline STR $(\%)$ & $76.7 \pm 5.1$ \\
\hline IO $(\%)$ & $72.7 \pm 5.0$ \\
\hline ALH $(\mu \mathrm{m})$ & $2.3 \pm 0.4$ \\
\hline BCF $(\mathrm{Hz})$ & $3.1 \pm 1.2$ \\
\hline
\end{tabular}

Los resultados se expresan como media \pm desviación estándar. MT: Movilidad total, MP: Movilidad progresiva, VCL: Velocidad curvilínea, VSL: Velocidad lineal, VAP: Velocidad media, LIN: Índice de linealidad, STR: Índice de rectitud, IO: Índice de oscilación, ALH: Amplitud lateral de la cabeza, BCF: Frecuencia de batida de la cola.

Los crioprotectores EG y DMSO tuvieron los mejores resultados post descongelación en las variables de movilidad total (62.1 y $59.7 \%)$ y progresiva (15.6 y $13.8 \%)$, respecto al resultado obtenido con DMF $(\mathrm{P} \leq 0.05)$. Las variables $\mathrm{VCL}, \mathrm{VSL}$, y $\mathrm{VAP}$ fueron superiores en el tratamiento con $\mathrm{EG}$, respecto a los demás crioprotectores ( $\mathrm{P} \leq 0.05)$ (Tabla 2), pero fueron inferiores a los valores obtenidos para el semen fresco. Los valores para LIN, STR e IO no presentaron diferencias entre los crioprotectores con semen post descongelado, pero fueron inferiores al semen fresco $(\mathrm{P} \leq 0.05)$. Finalmente, ALH y BCF no presentaron diferencias con semen fresco o criopreservado.

\section{Vitalidad espermática (VE)}

El crioprotector DMSO presentó vitalidad espermática superior, respecto a los crioprotectores DMF y EG $(\mathrm{P} \leq 0.05)$. La Tabla 3 presenta los resultados de VE para el semen fresco y post descongelado.

\section{Discusión}

Estos resultados corresponden al primer reporte sobre crioconservación y calidad espermática para la sabaleta Brycon henni, una especie promisoria para la acuicultura y la conservación de peces de importancia económica y social en Colombia, a pesar de la afectación en sus cuencas. Los efectos antrópicos han diezmado mucho sus poblaciones naturales, de allí el compromiso gubernamental para protegerla y realizar

Tabla 2. Resultados de calidad espermática post descongelación de semen de sabaleta Brycon henni, obtenidos mediante el sistema de análisis asistido por computador SCA ${ }^{\circledR}$.

\begin{tabular}{|l|c|c|c|}
\hline & DMF & DMSO & EG \\
\hline $\mathrm{n}$ & 38 & 42 & 36 \\
\hline $\mathrm{MT}(\%)$ & $52.7 \pm 9.2^{\mathrm{a}}$ & $59.7 \pm 9.3^{\mathrm{b}}$ & $62.1 \pm 11.2^{\mathrm{b}}$ \\
\hline $\mathrm{MP}(\%)$ & $11.7 \pm 6.7^{\mathrm{a}}$ & $13.8 \pm 6.8^{\mathrm{b}}$ & $15.6 \pm 7.8^{\mathrm{b}}$ \\
\hline $\mathrm{VCL}(\mu \mathrm{m} / \mathrm{seg})$ & $49.4 \pm 7.0^{\mathrm{a}}$ & $51.2 \pm 5.9^{\mathrm{b}}$ & $53.3 \pm 8.7^{\mathrm{c}}$ \\
\hline $\mathrm{VSL}(\mu \mathrm{m} / \mathrm{seg})$ & $23.8 \pm 4.4^{\mathrm{a}}$ & $24.4 \pm 4.1^{\mathrm{b}}$ & $25.6 \pm 5.4^{\mathrm{c}}$ \\
\hline $\mathrm{VAP}(\mu \mathrm{m} / \mathrm{seg})$ & $34.5 \pm 5.4^{\mathrm{a}}$ & $35.2 \pm 4.7^{\mathrm{b}}$ & $36.7 \pm 6.1^{\mathrm{c}}$ \\
\hline LIN $(\%)$ & $47.9 \pm 3.5^{\mathrm{a}}$ & $47.4 \pm 3.6^{\mathrm{a}}$ & $47.6 \pm 4.5^{\mathrm{a}}$ \\
\hline STR $(\%)$ & $68.4 \pm 3.1^{\mathrm{ab}}$ & $68.9 \pm 2.6^{\mathrm{abc}}$ & $69.4 \pm 3.0^{\mathrm{c}}$ \\
\hline $\mathrm{IO}(\%)$ & $69.8 \pm 2.8^{\mathrm{a}}$ & $68.9 \pm 3.6^{\mathrm{b}}$ & $68.9 \pm 4.0^{\mathrm{b}}$ \\
\hline ALH $(\mu \mathrm{m})$ & $2.6 \pm 0.7^{\mathrm{a}}$ & $2.4 \pm 0.2^{\mathrm{b}}$ & $2.5 \pm 0.3^{\mathrm{b}}$ \\
\hline BCF $(\mathrm{Hz})$ & $2.9 \pm 0.5^{\mathrm{a}}$ & $2.9 \pm 0.4^{\mathrm{a}}$ & $2.9 \pm 0.5^{\mathrm{a}}$ \\
\hline
\end{tabular}

Los resultados se expresan como media \pm desviación estándar. DMF: Dimetilformamida, DMSO: Dimetilsulfoxido, EG: Etilenglicol, MT: Movilidad total, MP: Movilidad progresiva, VCL: Velocidad curvilínea, VSL: Velocidad lineal, VAP: Velocidad media, LIN: Índice de linealidad, STR: Índice de rectitud, IO: Índice de oscilación, ALH: Amplitud lateral de la cabeza, BCF: Frecuencia de batida de la cola. Diferentes letras (filas) denotan diferencia estadística significativa $(\mathrm{P} \leq 0.05)$. 
Tabla 3. Resultados de vitalidad espermática en semen fresco y post descongelado de la sabaleta Brycon henni.

\begin{tabular}{|c|c|c|c|c|}
\hline & Fresco & DMF & DMSO & EG \\
\hline Media $\pm \mathrm{DE}$ & $90.7 \pm 4.3^{\mathrm{a}}$ & $73.5 \pm 5.9^{\mathrm{c}}$ & $82.0 \pm 1.9^{\mathrm{b}}$ & $69.2 \pm 6.7^{\mathrm{c}}$ \\
\hline
\end{tabular}

Los resultados se expresan como media \pm desviación estándar. Letras diferentes denotan diferencia estadística significativa $(P \leq 0.05)$.

un estudio de conservación genética, tanto en cautiverio como en su hábitat natural.

\section{Semen fresco y criopreservado}

Durante la época del estudio, los machos en cautiverio no fueron inducidos hormonalmente, presentando volúmenes variables de semen. Lo anterior se ajusta a lo reportado por Tabares et al., (2006), en donde el comportamiento reproductivo de los machos en cautiverio, podría obedecer más a los factores físico químicos del agua que a la influencia de la melatonina asociadas a la estación climática, por lo que producirían semen durante diez meses al año. Así mismo, no se presentaron diferencias en las características seminales entre lo obtenido en este trabajo y lo reportado por los mismos autores.

El valor de MT de semen fresco observado en sabaleta Brycon henni (96\%), fue superior al reportado por Tabares et al., (2004), en la misma especies (90\%), pero similar a otras especies de genero Brycon como $B$ orbygnianus (98\%) (Murgas et al., 2001), B insignis (92\%) (Andrade-Talmelli et al., 2001) y B amazonicus (96\%) (Cruz-Casallas et al., 2004). Por otro lado, considerando las variables de movilidad post descongelación, CruzCasallas et al., (2006), reportaron para B amazonicus, una MT de $76 \%$ y $60 \%$ con los crioprotectores DMSO $5 \%$ y EG 5\%, respectivamente. En el presente trabajo, se encontró que DMSO 5\% tuvo un porcentaje menor $(59.7 \%)$, lo cual podría sugerir un efecto de daño celular por desbalance entre la síntesis y utilización de ATP en los procesos metabólicos (Cruz-Casallas et al., 2006). Sin embargo, Carolsfeld et al., (2003) sugirieron que el DMSO 10\% proporciona los mejores resultados como crioprotector para los carácidos migratorios brasileros, entre los que se incluye el género Brycon. Respecto a DMSO y EG, no hubo diferencias significativas $(\mathrm{P} \geq 0.05)$, pero se presentó una tendencia a ser más

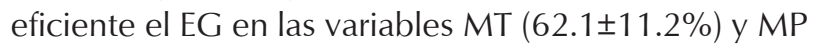

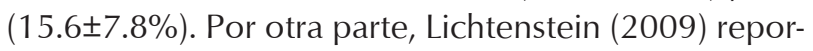
tó buenos resultados con DMSO 10\% y EG 10\% en la crioconservación de semen de Odonstesthes bonariensis, lo cual pone en evidencia la capacidad permeable de estos dos crioprotectores.
El crioprotector DMF presentó valores cercanos a los reportados para Colossoma macropomum (DMF 5\% = $55.7 \%$ ) (Varela-Junior et al., 2012), pero superiores a los descritos para Veraspes variegatus (DMF 13.3\% = 5\%) (Tian et al., 2008). Los resultados anteriores confirmaron, una vez más, que la crioconservación de semen en los peces, presenta resultados altamente variables y que el proceso es muy sensible a diferentes factores, siendo necesario realizar ajustes específicos a los protocolos para cada especie (Medina et al., 2005; Martínez, 2008).

Las demás variables obtenidas con el sistema computarizado, presentaron una tendencia superior para el EG, más no significativa frente a DMSO. Lo anterior sugiere una mayor exploración de estos crioprotectores en su desempeño post descongelación en aspectos como la tasa de fertilización, tiempo de crioconservación, criodaño célular y en el ADN.

\section{Vitalidad}

La vitalidad presentó un mayor porcentaje para DMSO $(82.0 \pm 1.9 \%)$, lo cual sugiere un menor daño en la integridad de la membrana, solo un $10 \%$, con el uso de este crioprotector respecto al semen fresco. De igual manera, Vuthiphandchai et al., (2009) reportaron un alto porcentaje (92.7\%) de vitalidad en Lutjanus argentimaculatus con DMSO $10 \%$, confirmándose la preferencia del DMSO en la mayoría de los procesos de crioconservación de semen de peces. La evaluación de este parámetro permite la predicción de la fertilidad potencial en muestras de semen, ya que una membrana plasmática intacta es necesaria para la fecundación (Chalah y Brillard, 1998).

En conclusión, el uso de los crioprotectores DMSO y EG presentaron porcentajes superiores de MT y MP, con una ligera tendencia superior de EG en las demás variables. Así mismo, DMSO presentó los mejores porcentajes de vitalidad, con el semen post descongelado de sabaleta Brycon henni.

\section{Agradecimientos}

A la Coordinación de Investigación y Posgrado por el apoyo financiero. Al técnico profesional Jorge Eliecer 
Rodríguez Rojas, por la labor logística, a William Berrio y María Trinidad Chica por su colaboración administrativa, en la Granja Experimental y de Producción Piscícola "John Jairo González Torres", del Politécnico Colombiano Jaime Isaza Cadavid.

\section{Referencias}

Andrade-Talmelli E, Kayamoto E, Fenerich-Verani N. Características seminais da piabanha Brycon insignis (Steindachner, 1876) após estimulação hormonal. Biol Inst Pesca, São Paulo. 2001;27(2):149-154.

Betancur J, Ospina J, Botero J, Uribe J, Botero M, Montoya A. Efecto de diferentes activadores espermáticos sobre el porcentaje de movilidad y tiempo de activación en semen de dorada Brycon moorei (Dahl, 1955) fresco y criopreservado. Rev Colomb Cienc Pecu. 2009;22:385.

Carolsfeld J, Godinho H, Zaniboni-Filho E, Harvey B. Cryopreservation of sperm in Brazilian migratory fish conservation. J Fish Biol. 2003;63:472-189.

Challah T, Brillard J. Comparison of assessment of fowl sperm viability by Eosin Nigrosin and dual fluorescence (SYBR-14/PI). Theriogenology. 1998;50:487-493.

Cruz-Casallas P, Pardo-Carrasco S, Arias-Castellanos J, Lombo-Castellanos P, Lombo-Rodriguez D. Cryopreservation of yamu Brycon siebenthalae milt. J World Aquacult Soc. 2004;35:529535.

Cruz-Casallas P, Medina V, Velasco Y. Evaluación de diferentes crioprotectores para la crioconservación de espermatozoides de yamú Brycon amazonicus. Rev Colom Cien Pec. 2006;19(2):153-159.

Dahl G. 1971. Los peces del norte de Colombia. Bogotá (Colombia): Instituto de Desarrollo de los Recursos Naturales Renovables, (Inderena), Talleres Litografia Arco. 391p.

Fresneda A, Lenis G, Agudelo E, Olivera M. Espermiación inducida y crioconservación de semen de cachama blanca Piaractus brachypomus. Rev Colom Cien Pec. 2004;17:46-52.

Gonzalez J, Losada E, Cruz-Casallas N, Cruz-Casallas P, Medina V. Efecto de la sustancia crioprotectora y nivel de glucosa sobre la viabilidad de embriones de cachama blanca Piaractus brachypomus conservados a $-14{ }^{\circ} \mathrm{C}$. Rev Colomb Cienc Pecu. $2011 ; 24: 384$.

Gillan L, Evans G, Maxwell W. Flow cytometric evaluation of sperm parameters in relation to fertility potential. Theriogenology. 2005;63(2):445-457.

Hurtado J, Mancera N, Saldamando C. Variabilidad genética de Brycon henni (Characiformes: Characidae) en la cuenca media de los ríos Nare y Guatapé, sistema río Magdalena, Colombia. Rev Biol Trop. 2011;59:269-282.

Instituto Colombiano Agropecuario (ICA). 2007. Buenas prácticas en la producción acuícola: directrices sanitarias y de inocuidad para la producción acuícola destinada al consumo humano. Subgerencia de Protección y Regulación Pecuaria. Grupo de Inocuidad en las Cadenas Agroalimentarias Pecuarias. Grupo de prevención y análisis de riesgos zoosanitarios y asuntos internacionales. Produmedios. Bogotá. 67p.

Lenis G, Restrepo L, Rivera J, Monsalve F, Cruz-Casallas P. Reproducción inducida y producción de alevinos de Sabaleta Brycon henni: determinación del tiempo de latencia utilizando extracto de hipófisis de carpa. Rev Colom Cien Pec. 2009;22:143-155.

Lichtenstein G. 2009. Desarrollo e implementación de técnicas para la crioconservación de esperma de pejerrey bonaerense Odonstesthes bonariensis. Facultad de Ciencias Exactas y Naturales. Universidad de Belgrano.

Martínez S. 2008. Bases para la elaboración de bancos de germoplasma en peces: aplicación a la trucha leonesa. Facultad de Ciencias Biológicas y Ambientales. Departamento de Biología Molecular. Universidad de León. 174p.

Martinez G, Atencio V, Pardo S. Efectos de la concentración de glucosa sobre la activación de la movilidad espermática en bocachico Prochilodus magdalenae (Pisces, Characiformes) Rev MVZ Córdoba. 2011;16(2):2554-2563.

Martínez J, Pardo-Carrasco S, Tarazona-Morales A. Sperm cryopreservation of freshwater fish bocachico Prochilodus magdalenae in DMSO and glucose and its effects on fertilization and hatching efficiency. Anim Reprod. 2012;9:19-26.

Medina M, Velasco Y, Cruz-Casallas P. Aspectos generales de la criopreservación espermática de peces teleósteos. Rev Colom Cien Pec. 2005;18(1):34-36.

Montoya A, Carrillo L, Olivera M. Algunos aspectos biológicos y del manejo en cautiverio de la Sabaleta Brycon henni Eigenmann, 1913 (Pisces: Characidae) Rev Col Cienc Pec. 2006;19(2):180185.

Murgas L, Gualhanone A, Silva M, Mello C, Freitas R, Zangeronimo M. Calidad seminal del pez pirancanjuba Brycon orbignyanus post descongelación. An Vet. (Murcia). 2001;17:3-10.

Navarro O, Velasco Y, Cruz-Casallas P. Evaluación de cinco protectores para la crioconservación de semen de Cachama Blanca Piaractus brachypomus, Rev Col Cienc Pec. 2004;17:53-59.

Pardo-Carrasco S, Zaniboni-Filho E, Arias-Castellanos J, Suarez-Mahecha $\mathrm{H}$, Atencio-García V, Cruz-Casallas P. Evaluation of milt quality of the yamú Brycon amazonicus under hormonal induction. Rev Colom Cienc Pec. 2006;19:134-139.

Pineda-Santis H, Arboleda L, Echeverry A, Urcuqui S, Pareja D, Olivera $M$, Builes J. Caracterización de la diversidad genética en el pez Brycon henni (Characiformes: Characidae) en Colombia central por medio de marcadores RAPD. Rev Biol Trop. 2007;55:1025-1035.

Pinzón-Arciniegas S, Mojica-Rodríguez J; Cruz-Casallas P. Ensayos preliminares sobre crioconservación de semen de bagre rayado (Pseudoplatystoma fasciatum Linnaeus, 1766). Orinoquia. 2005;9:28-37.

Ramirez J, Medina V, Cruz-Casallas P. Crioconservación de semen de bagre rayado Pseudoplatystoma fasciatum (Linnaeus, 1766) empleando un congelador programable: evaluación de la movilidad y fertilidad post descongenlación. Rev Colomb Cienc Pecu. 2008;21:473. 
Ramalho-Santos J, Amaral A, Sousa A, Rodrigues A, Martins L, Baptista M, Mota P, Tavares R, Amaral S, Gamboa S. 2007. Probing the structure and function of mammalian sperm using optical and fluorescence microscopy. Modern Research and Educational Topics in Microscopy 394-402.

Restrepo G, Ocampo D, Velasquez A. Evaluación de la movilidad del semen criopreservado de caballos criollo colombiano por un Sistema analizador de clase. Revista UDCA. 2013;16(2):445450.

Reza L, Salas J. 2010. Crioconservación de semen de bagre blanco Sorubim cuspicaudus (Littmann, Burr \& Nass 2000) con Dimetilacetamida (DMA) y Dimetilsulfoxido (DMSO). Facultad de Medicina Veterinaria y Zootecnia. Programa de Acuicultura. Universidad de Córdoba. 60p.

Tabares J, Montoya A, Ruiz Z, Cerón M, Arboleda L, Olivera M. Caracterización seminal en sabaleta Brycon henni: evaluación de factores inhibidores y activadores de la motilidad espermática. latreia. 2004;17(2):173-174.

Tabares J, 2005. Evaluación del efecto de algunos iones sobre la activación de la movilidad espermática y el potencial de membrana en Brycon henni, tesis, Facultad de Ciencias Agrarias, Universidad de Antioquia, Medellín; 68p

Tabares J, Montoya A., Arboleda L, Echeverri A, Restrepo L, Olivera A. Efecto de la pluviosidad y el brillo solar sobre la producción y características del semen en el pez (Brycon henni) Rev Biol Trop. 2006;54(1):179-187.

Tabares J, Ruiz T, Arboleda L, Olivera A. Effect of some ions on sperm activation in Brycon henni. Efecto de algunos iones sobre la activación espermática en Brycon henni. Acta Biol Colom. 2007; 12:87-98.

Tian Y, Chen S, Ji X, Zhai J, Sun L, Chen C, Su P. Cryopreservation of spotted halibut (Veraspes variegatus) sperm. Aquaculture. 2008;284:268-271.

Tiersch T. Strategies for commercialization of cryopreserved fish semen. R Bras Zootec. 2008;37:15-19.

Varela-Junior A, Corcini C, Gheller S, Jardim S, Lucia-Junior C, StreitJunior D, Figueiredo M. Use of amides as cryoprotectants in extenders for frozen sperm for tambaqui Colossoma macropoum. Theriogenology. 2012;78:244-251.

Viveiros ATM, Godinho HP Sperm quality and cryopreservation of Brazilian freshwater fish species: a review. Fish Phys Bioch. 2009;35:137-150.

Vuthiphandchai V, Chomphuthawach S, Nimrat S. Cryopreservation of red snapper (Lutjanus argentimaculatus) sper: effect of cryoprotectans and cooling rates on sperm motility, sperm viability and fertilization capacity. Theriogenology. 2009;72:129-138. 